\title{
Non Selective Inhibition of COX Activity Reversed Inflammation and Reactive Oxygen Radicals Mediated Prostate Cancer Risk and Decreased Disease Progression in Preclinical Model
}

\author{
Maxwell Omabe1,2*, Kenneth Nwobini Omabe ${ }^{3}$, Clement Ademola Famurewa ${ }^{4}$, \\ Alberta Egwu Okorocha5, Grace Maxwell Omabe ${ }^{2}$ \\ ${ }^{1}$ Tumor Immunology Group, Division of Oncology, Cancer Research Division, Department of Pathology and \\ Laboratory Medicine, University of Saskatchewan, Saskatoon, Canada \\ ${ }^{2}$ Molecular Pathology and Cancer Biology Research Group, Chemical Pathology Division, Department of \\ Medical Laboratory Sciences, Faculty of Health Science, Ebonyi State University, Ebonyi, Nigeria \\ ${ }^{3}$ Department of Biochemistry and Molecular Biology, Faculty of Natural Sciences, Federal University, Ndufu \\ Alike Ikwo, Nigeria \\ ${ }^{4}$ Department of Medical Biochemistry, Faculty of Basic Medical Sciences, Federal University, Ndufu Alike Ikwo, \\ Nigeria \\ ${ }^{5}$ Department of Physiology, Faculty of Basic Medical Sciences, Ebonyi State University, Ebonyi, Nigeria \\ Email: *Maswello2002@yahoo.com
}

Received 12 November 2015; accepted 25 January 2016; published 28 January 2016

Copyright (C) 2016 by authors and Scientific Research Publishing Inc.

This work is licensed under the Creative Commons Attribution International License (CC BY).

http://creativecommons.org/licenses/by/4.0/

(c) (i) Open Access

\section{Abstract}

Prostate cancer (PCa) represents the most frequent urologic diagnosis in elderly males. We have previously shown that exposure of prostate to lipopolysaccharide (LPS) promotes cancer risk. We investigated the effect of non-selective cyclooxygenase (COX) inhibition on prostate inflammation-mediated cancer risk in vivo. The prostates of male rats were inoculated with $E$. coli as sources of inflammatory molecules (LPS) and were treated with COX inhibitor, aspirin $2 \mathrm{mg} / \mathrm{Kg}$ orally for 14 days or PBS. Oxidative stress was induced with two $2 \mathrm{mls}$ of hydrogen peroxide orally twice daily or PBS for 14 days; they were either treated with COX inhibitor or PBS for another 14 days. Blood was collected and analyzed for acid phosphatase and PSA. Data showed presences of LPS in the prostate of the rats resulted in gradual increase in PSA when compared to control $(P<$ 0.0001). However, COX inhibition resulted in statistically significant reduction in concentration of

\footnotetext{
${ }^{*}$ Corresponding author.
}

How to cite this paper: Omabe, M., et al. (2016) Non Selective Inhibition of COX Activity Reversed Inflammation and Reactive Oxygen Radicals Mediated Prostate Cancer Risk and Decreased Disease Progression in Preclinical Model. Journal of Cancer Therapy, 7, 41-49. http://dx.doi.org/10.4236/jct.2016.71005 
PSA level compared to control group $(P<0.0001)$. To understand if oxidative stress mechanism was involved in the inflammation mediated increase in PSA, data showed that rats exposed to $\mathrm{H}_{2} \mathrm{O}_{2}$ had 2.5 fold increase in acid phosphatase (ACP) compared control $(P<0.0001)$, and by inhiting COX activity, a statistically significant reduction in ACP from $11.2 \mathrm{IU} / \mathrm{L} \pm 0.67$ to $5.7 \mathrm{IU} / \mathrm{L} \pm 0.347(P<$ 0.0034) was observed. Thus since increased in PSA was associated to cancer risk, our data suggested that inflammation mediated prostate cancer risk was reversible by Inhibition of COX Activity in rats.

\section{Keywords}

Inflammation, Prostate Cancer, Reactive Oxygen Species

\section{Introduction}

Prostate cancer ( $\mathrm{PCa}$ ) is the most common cancer in the adult male, and benign prostatic hyperplasia (BPH) represents the most frequent urologic diagnosis in elderly males [1]. Recent data suggest that prostatic inflammation is involved in the pathogenesis and progression of both conditions [2] [3]. The observation of chronic inflammation coexisting with BPH histologic changes in pathologic specimens leads to the suspicion that inflammation may play a role in the development of prostate enlargement. Prostatic inflammation is associated with overall clinical progression [4].

BPH and PCa form in different areas of the prostate [5]. The former is known to develop from the transitional zone (TZ) and central zone of the gland, while the latter develops from the peripheral zone (PZ). Only in about $20 \%$ of cases do the conditions coexist in the same zone [5]. BPH and PCa are considered chronic diseases, with early initiation and slow progression [6]. BPH starts as a simple micronodular hyperplasia, evolving into a macroscopic nodular enlargement that gradually translates into a clinical entity [7]. Similarly, PCa develops through early and late precancerous histologic modifications [4]. Furthermore, although there is no clear molecular and genetic relationship between BPH and PCa, they present two distinct pathogenetic pathways, epidemiologic studies suggest that because their incidence and prevalence rise with increased age, both conditions are hormone dependent and are associated with prostatic inflammation, which can represent a common denominator [1] [8].

Local inflammation may be triggered by a viral or bacterial infection or other pathogen associated molecular pattern (PAMP), which will lead to the secretion of cytokines, chemokines, and growth factors involved in the inflammatory response with consequent growth or proliferation of epithelial and stromal prostatic cells [4] [6].

The atypical chemokine receptor D6 is a decoy and scavenger receptor for most inflammatory CC chemokines [9]. In fact, the appropriate control of the chemokine system involves several chemokine decoy receptors, with distinct specificity and tissue distribution, and controls most inflammatory CC chemokines [9]. In health, the D6, has been shown to abrogate CC chemokines which are known to mediate inflammatory response; by this shut off inflammation by making the chemokines unavailable [9] [10]. For example, evidence from experimental studies demonstrates that mice deficient of D6 has increased susceptibility to develop cancer induced by the inflammation-producing carcinogen, the phorbol ester [10]. In contrast, transgenic mice with lots of D6 are resistant to tumor formation [10]. This strongly suggests that inflammation can be protumorigenic. We have previously shown that infection and inflammation are major confounding factors in the pathogenesis of prostate cancer [3]. We have recently shown that exposure of the prostate to molecules that trigger chronic inflammation promotes the risk for prostate carcinogenesis [11]. Here, we investigate the role of cyclooxygenase inhibitor on prostatic epithelium with inflammatory stimulus and an altered physiologic response due to a change in the prostatic microenvironment. We show that cyclooxygenase COX inhibition suppresses acid phosphatase (ACP) levels and results in decreased level of pre-treatment lipopolysaccharide (LPS) mediated increased in prostate specific antigen (PSA) in rats.

\section{Material and Method}

\subsection{Animal Experiment}

Adult male rat weighing between 130 - 220 g were used for the study, the animals were obtained from animal house at Enugu State University, department of animal science. On arrival, the animals were placed at random 
and allocated for treatment in a wire gauze cage with paddy husk as bedding. Animals were housed at a temperature of $24^{\circ} \mathrm{C} \pm 3^{\circ} \mathrm{C}$ in Ebonyi State University research laboratory where all the animals were allowed to free access to fed and water (with standard commercial polluted rat chaw) without any treatment for 2 weeks, this is to allow them to acclimatize. All chemicals and reagents purchased and used for these studies were analytical grade (Sigma, Nigeria) and ABL Nigeria.

\subsection{Experimental Prostatitis with Lipopolysaccharide}

The weight of the animals was measured for both test and control. The method De Marzo et al. 2007 was used. In detail, pure colonies of E. coli measuring $1 \times 10^{5}$ (obtained from Federal teaching hospital Abakaliki) was emulsified to a concentration of $1 \times 10^{4}$ of PBS in a plain container and was allowed to completely dissolve. A 2 $\mathrm{ml}$ syringe (ABL, Nigeria) was used to inject directly into the prostate of the rat $0.2 \mathrm{ml}$ of the bacteria solution. The animals were allowed free access to food and water both test and control. The control animals were treated same as the test but with normal saline only for control group. The animals were closely observed on daily bases and were carefully monitored for at least 6 days. The animals' weights were measured and recorded. All animals were handled as specified by ethical committee for animal right. At least $2 \mathrm{ml}$ of blood was collected from each rat by cardiac puncture for both the test and control into a plain (anticoagulant free) container and allowed to clot following a standard protocol. Then those samples were centrifuged at $3000 \mathrm{~g}$ per minute at room temperature. The serum was separated and stored in the freezer at $-20^{\circ} \mathrm{C}$ till when required for analysis.

\subsection{Treatment with Hydrogen Peroxide}

Oxidative stress was induced in the rats by oral administration of $3 \%$ hydrogen peroxide according to published protocol [11]. In detail, the rats were randomized into 2 groups and were treated with 2 mls of hydrogen peroxide orally twice daily or PBS for 14 days. Another group had similar treatment as the later, but was treated with aspirin (COX) $2 \mathrm{mg} / \mathrm{Kg}$ or PBS twice daily for 14 days, while their weights were also measured daily. The two groups were monitored for at least 14 days. The blood samples were collected and biochemical studies were undertaken for ACP and PSA.

\subsection{Treatment with COX Inhibitor}

The rats previously exposed to local prostatic inflammatory stimulus through exposure with LPS from $E$. coli.. They were then divided into 2 groups. The test group was treated with $2 \mathrm{mg} / \mathrm{kg}$ of aspirin orally 2 times daily for 8 days, while the control group received vehicle of PBS for the same duration, and blood samples were collected and assessed.

\subsection{Laboratory Analysis}

Prior to the time of analysis, the samples were allowed to return to room temperature by removing the samples from the refrigerator. Each serum sample from the two separate experiments was analyzed for "prostate specific antigen (PSA) using enzyme immunoassay with microtitre wells (Dako, USA). The principle of the ELISA test was based on solid phase enzyme linked immunosorbent assay technique. The assay system utilizes a goat antiPSA antibody directed against PSA for solid phase immobilization (using the microtiter plate). A monoclonal anti-PSA antibody directed against PSA antigen. A monoclonal anti-PSA antibody conjugated to horseradish peroxidase (HRP) was in the antibody-enzyme conjugate solution. The test was performed according to the manufacturer's instruction.

The test samples were allowed to react first with the immobilized goat antibody at room temperature for 60 minutes. The wells were washed to remove any unbounded antigen. The monoclonal anti-PSA-HRP conjugate was reacted with the immobilized antigen for 60 minutes at room temperature, resulting in the PSA molecules being sandwiched between the solid phase and enzyme linked antibodies. The wells were washed with water to remove unbound labeled antibodies. A solution of TMB reagent was added and incubated at room temperature for 20 minutes, resulting in development of a blue color. Color development is stopped with the addition of stop solution changing the color to yellow. The concentration of PSA is directly proportional to the color intensity of the test sample. Absorbance is measured spectrophotometrically at $450 \mathrm{~nm}$ wavelength. 


\subsection{Measurement of Prostate Specific Acid Phosphatase}

Serum level of acid phosphotase was measured in accordance with published method [12]. In brief, total and prostate specific acid phosphotase which were determined according to established protocol. P-Nitrophenyl phosphate is hydrolyzed in acid $\mathrm{pH}$ medium by the action of acid phosphatase present in serum. The liberated p-Nitrophenol was quantified spectrophotometrically. The tartaric acid acts as inhibitor of the prostatic acid phosphatase. This tartaric-sensitive fraction was measured by the difference in activity observed when the assay was carried out in the absence and presence of tartaric acid.

\subsection{Statistical Analysis}

The statistical analyses was performed using Prism software. For analysis of PSA, the values were multiplied by 10 in other to get whole numbers. The results are expressed as mean \pm SEM, $(N=3)$. Test of statistical significance was determined by one-way analysis of variance with $\mathrm{P}<0.05$ considered significant. Statistical analysis of the result obtained was done using mean \pm standard error of mean (S.E.M.).

\section{Result}

\subsection{Effect of Chronic Local E. coli and Lipopolysaccharide (LPS) on the Prostate}

Figure 1 shows the PSA level of test rat with or without exposure of the prostate to LPS and development of experimental prostatitis compared to the control group. There was a gradual increase in serum PSA when compared to the control; resulting in increase in level of PSA which was statistically significant at $95 \%$ confidence interval $(P<0.0001)$; suggesting that infection of the prostate epithelium and exposure to the highly inflammatory molecule, LPS may mediate state of local epithelial inflammatory which interfere with the epithelial cell physiology and result in increased PSA secretion in rat.

\subsection{Oxidative Stress Results in Increase in Tartrate Sensitive Prostate Acid Phosphatase (ACP) Concentration}

Abundantly published studies have shown that inflammation sustained by infection, produces simple epithelial hyperplasia, increased epithelial cell proliferation, through an oxidative stress. To investigate this, animals were induced to have oxidative stress from generated reactive oxygen species after treatment with hydrogen peroxide. The prostate specific acid phosphatase levels were determined. Data showed a 2.5 fold increase in acid phosphatase in the rats that were treated with hydrogen peroxide compared to the control group $(P<0.0001)$ (Figure 2).

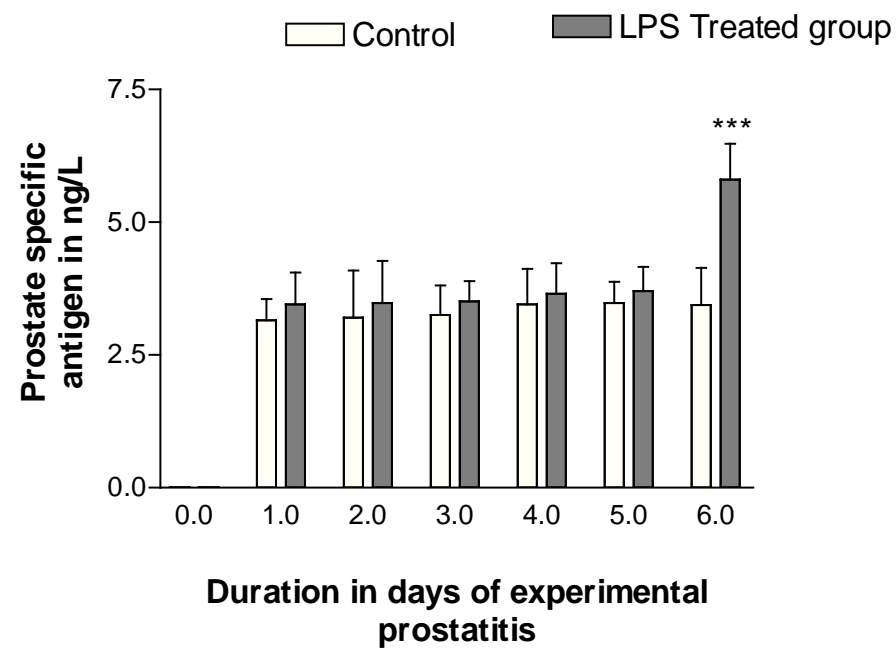

Figure 1. Illustrates the gradual increase in serum PSA levels in experimentally induced prostate infection in immunocompetent mice when compare to the control group. PSA level were determined as described in the materials and method and transformed by multiplying with 10. Data were compared with the untreated group. The experiment was repeated 3 times and the SEM determined. $\mathrm{n}=3$. Error bar represent standard error of mean. ${ }^{* * *}=P<0.0001$. 


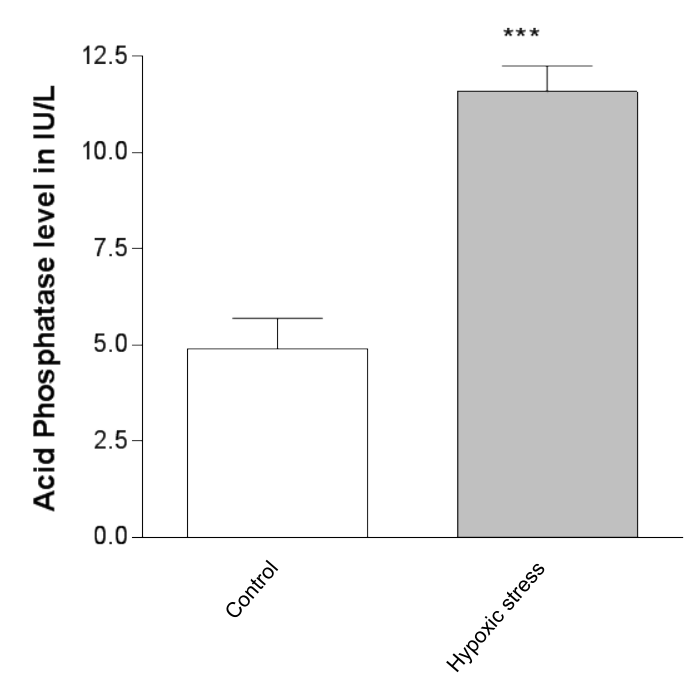

A preclinical study involving rats expsed to hypoxic stress and controls 14 days. Data show mean and SEM. $* * *=\mathrm{P}<0.0001$

Figure 2. Adult male rats were experimentally induced to hypoxic stress by daily treatment with hydrogen peroxide and the control group received only PBS for 7 days. Data showed that exposure oxidative stress resulted in $\mathrm{m} 2.5$ folds increase in prostate specific acid phosphatase compared to the control, demonstrating a statistically significant $(P<0.0001)$. Data represents mean and error bar $=\mathrm{SEM}$.

\subsection{Inhibition of COX Activity Reversed Inflammation Mediated Increase in PSA or ACP}

A feature of oxidative stress reactions is the production of arachidonic acid from membranes, a process associated with the generation of reactive oxygen radicals, causing oxidative damage, as well as being converted by the cyclo-oxygenase (COXs) to various eicosanoids and prostaglandins, which are important factors in the regulation of cell proliferation (Alessandro et al. 2008). To test this COX activity was inhibited in the rats previously exposed to LPS or oxidative stress using hydrogen peroxide, and ACP and PSA were determined. Data showed that treatment with non selective COX inhibitor for 14 days caused statistically significant decrease in PSA elevation $(P<0.0001)$ compared to the control group (Figure 3), and resulted in statistically significant reduction in ACP level from 11.2 IU/L \pm 0.67 to $5.7 \mathrm{IU} / \mathrm{L} \pm 0.347(P<0.0034)$, and a negative correlation of $\mathrm{R}^{2}=0.73$ (Figure 3 and Figure 4).

\subsection{Oxidative Stress Results in Insignificant Increase in Weight}

Figure 5 shows the relationship in weight of rats that received daily doses of hydrogen peroxide and the group that received PBS as vehicle. Data show that there was no significant difference in the weight of the rats that were exposed to oxidative stress.

\section{Discussion}

Data from this study confirm previous reports from our laboratory [3] [11], and others [13]-[15]; which earlier suggest that prostatitic infection or inflammation may be associated with disease progression and more risk for prostate tumour initiation. A common feature of this form of cancer is prostate pathology with characteristic increased PSA level.

We have shown that the presence of highly inflammatory molecule LPS in the prostate results in statistically significant increase in PSA level in rats (Figure 1). To assess if the inflammation mediated increase in PSA is associated with signaling through oxidative or hypoxia pathways, Figure 2 shows that whenever the rats are induced to hypoxic stress to generate increasing level of reactive oxygen species, it is found that there is a 


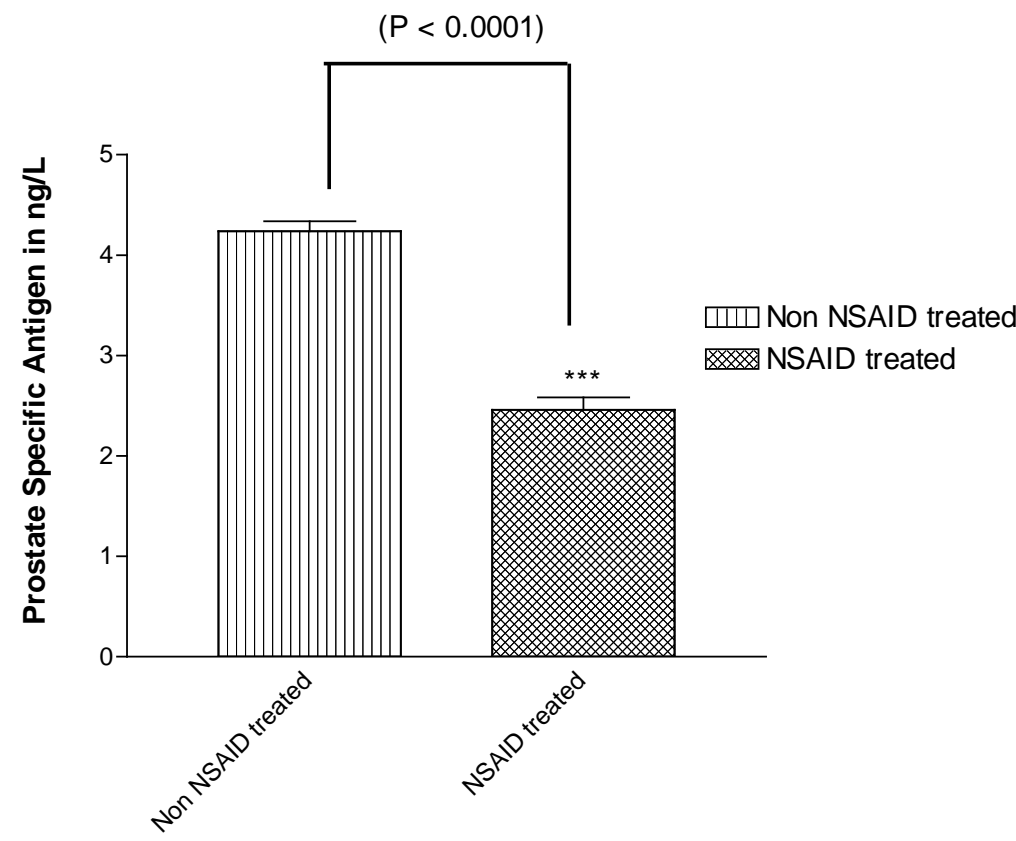

Figure 3. Rats previously exposed to local prostatic LPS received non selective COX inhibior $2 \mathrm{mg}$ per body weight twice daily for 14 days, while the control group received PBS only for same number of days. The experiment was repeated 3 times and the SEM determined. $\mathrm{n}=3$. Error bar represent standard error of mean. ${ }^{* * *}=P<0.0001$.

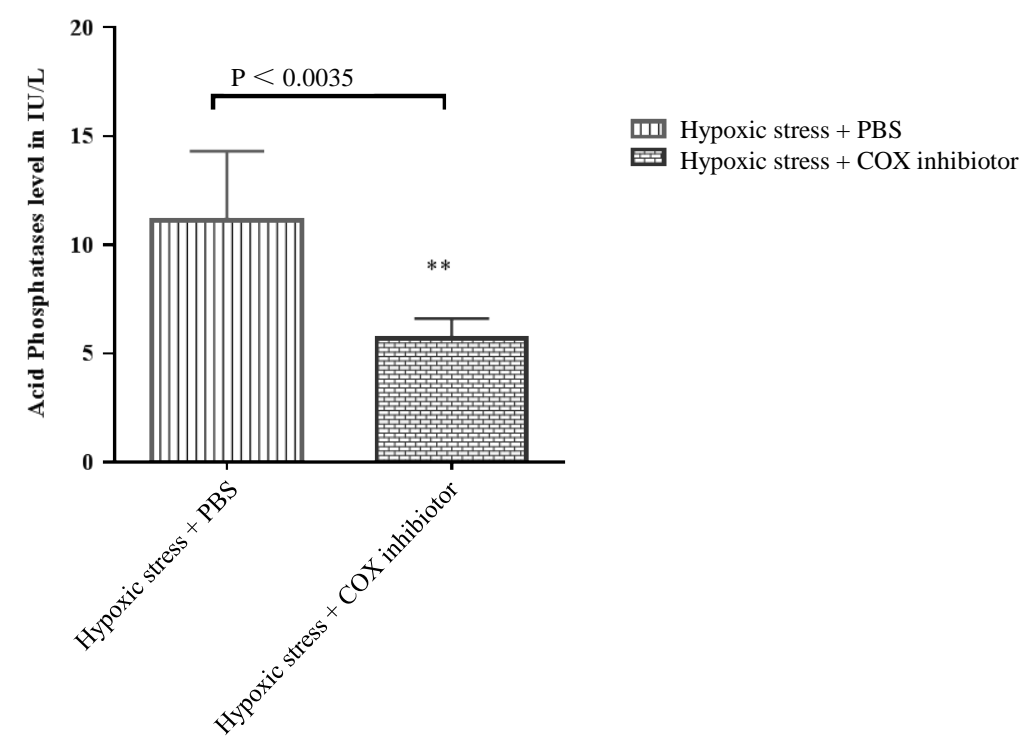

Figure 4. Treatment with non selective COX inhibitor statistically reduced ACP level from. The experiment was repeated 3 times and the SEM determined. $n=3$. Error bar represent standard error of mean. ${ }^{* *}=P<0.0035$

corresponding increase in the concentration of serum tartrate sensitive prostate specific acid phosphatase $(P<$ 0.0001). In tandem, the weight of the animals surprisingly does not decrease, however, the marginal but statistically insignificant increase in the weight (Figure 5) of the hypoxia exposed animals can be attributed to endocrine response to hypoxic stressor. Although we do not understand why weight is not altered, however, it is generally accepted that stress can cause metabolic alterations resulting in initial insulin resistance and increase in some acute phase proteins levels; the extend and duration of which determines if weight will be altered. Evidence 


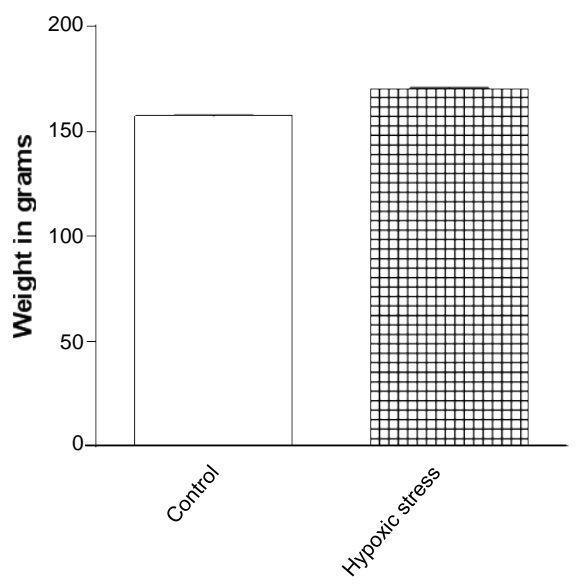

A preclinical study involving rats expsed to hypoxic stress and controls for at least 14 days

Figure 5. Represents the mean weight after day 7 the animals were treated with hydrogen peroxide 2 drops twice daily for 14 days and the controls received PBS 2 drops twice daily. The experiment was repeated 3 times and the SEM determined. $n=3$. Error bar represent standard error of mean.

has shown that cortisol which is a known response hormone to both inflammation and stress functions to induce perturbation of carbohydrate metabolism and cause insulin resistance [16]. This may in part explain the marginal increase in weight found in the hypoxia treated animals.

These data confirm a possible association between inflammation and the development of prostate disease and prostate cancer risk. In line with the current study, Dennis et al. [17] examined the consistency of the associations between prostatitis and PC; using a meta-analysis, the author found that the association between prostatitis and PC was significant among the population-based, case control studies $(\mathrm{OR}=1.8)$ and overall $(\mathrm{OR}=1.6)$.

To investigate the role of oxidative stress in this process, oxidative stress is induced, after treatment with hydrogen peroxide. Figure 3 demonstrate that reactive oxygen species and oxidative stress promote increase in tartrate sensitive prostate specific acid phosphatase significantly. Our data confirms a previous report and suggests that inflammation and oxidative stress or hypoxia can produce result in epithelial hyperplasia, increase epithelial cell proliferation [18]; marked by increased in both PSA and tatrate sensitive acid phosphatase level in rat.

It is known that a characteristic feature of oxidative stress reactions is the production of arachidonic acid from membranes; a process associated with the generation of reactive oxygen radicals and converted by the cyclooxygenase (COXs) to various eicosanoids [19], in particular prostaglandins, important factors in the regulation of cell proliferation [20]. We investigate whether this process also contributes to abnormal high level of ACP or PSA recorded in this study. We show that inhibition with non-selective COX activity but not with PBS significantly represses, reverses and returns the concentration of ACP $(P<0.0035)$ or PSA level, allowing it to drop to near baseline $(P<0.0001)$ when compared with the control group. In fact there is a strong correlation between non selective inhibition of COX and serum concentration of tartrate sensitive prostate specific ACP $\left(R^{2}=0.73\right)$.

The findings from this study suggest that prostatic oxidative stress [21]-[23], and LPS mediated inflammation [11], can elicit biochemical increase in biochemical indicators of prostate hyperplasia [24] and risk of prostate cancer [25]. COX activity has been shown to play an important role in the association between inflammation and prostate growth leading to generation of reactive oxygen species (ROS) and genomic damage [18]. Therefore, we have shown that by inhibiting COX activity, inflammation and oxidative stress mediated or ROS mediated increased in biochemical markers of prostate hyperplasia and prostate pathology [26] [27] including PSA and ACP are repressed in rat models. Concisely, pathogen associated molecular pattern including LPS are potent inducers of inflammatory mediated prostate cancer risk, we have shown that non selective inhibition of COX activity abrogated prostatic cell malignant-like behavior in murine model. COX inhibitors act downstream the 
arachnidonic acid metabolism; hence by inhibiting the cyclooxygenase pathway, the increased secretion of ACP or PSA was dramatically shutdown, confirming that inflammation and ROS generation contribute in part to promote transformation to prostate cancer. Data also confirmed that inflammation mediated changes in the physiology and prostate microenvironment, is reversible and preventable by blocking of the Cox signaling pathway production. The implication of these finding is that prostate disease may be preventable.

\section{Submission Declaration}

The authors declare that no part of this work has been published elsewhere or is awaiting publication anywhere.

\section{Conflict of Interest}

I declare no conflict of interest, and that no fund from any funding body or the University was made available for this work. This study was funded by the authors only.

\section{References}

[1] Maitland, N.J. and Collins, A.T. (2008) Prostate Cancer Stem Cells: A New Target for Therapy. Journal of Clinical Oncology, 10, 2862-2870.

[2] De Marzo, A.M., Platz, E.A, Sutchliffe, S., Xu, J.F., et al. (2007) Inflammation in Prostate Carcinogenesis. Nature Review Cancer, 7, 256-269. http://dx.doi.org/10.1038/nrc2090

[3] Omabe, M. and Ezeani, M. (2011) Infection, Inflammation and Prostate Carcinogenesis. Infection. Journal of Infection, Genetics and Evolution, 11, 1195-1198. http://dx.doi.org/10.1016/j.meegid.2011.03.002

[4] Sfanos, K.S. and De Marzo, A.M. (2012) Prostate Cancer and Inflammation: The Evidence. Histopathology, 60, 199-215. http://dx.doi.org/10.1111/j.1365-2559.2011.04033.x

[5] Omabe, M., Ewenighi, C., Ezeani, M., et al. (2011) Tumor Hypoxia and Treatment Resistant Prostate Cancer. Journal of Medicine and Medical Sciences, 2, 1306-1312.

[6] Vral, A., Magri, V., Montanari, E., et al. (2012) Topographic and Quantitative Relationship between Prostate Inflammation, Proliferative Inflammatory Atrophy and Low-Grade Prostate Intraepithelial Neoplasia: A Biopsy Study in Chronic Prostatitis Patients. International Journal of Oncology, 41, 1950-1958.

[7] Che, M. and Grignon, D. (2002) Pathology of Prostate Cancer. Cancer and Metastasis Reviews, 21, 381-395. http://dx.doi.org/10.1023/A:1021242813374

[8] Palapattu, G.S., Sutchiffe, S., Bastian, P.J., et al. (2005) Prostate Carcinogenesis and Inflammation Emerging Insights. Carcinogenesis, 26, 1170-1181. http://dx.doi.org/10.1093/carcin/bgh317

[9] Aoi, J., Endo, M., Kadomatsu, T., et al. (2011) Angiopoietin-Like Protein 2 Is an Important Facilitator of Inflammatory Carcinogenesis and Metastasis. Cancer Research, 71, 7502-7512. http://dx.doi.org/10.1158/0008-5472.CAN-11-1758

[10] Nibbs, R.J., Gilchrist, D.S., King, V., et al. (2007) The Atypical Chemokine Receptor D6 Suppresses the Development of Chemically Induced Skin Tumors. Journal of Clinical Investigation, 117, 1884-1892. http://dx.doi.org/10.1172/JCI30068

[11] Omabe, M., Omabe, K., Okwuegbu, M., Grace, O. and Okoro, D.U. (2014) Exposure of Prostate to Lipopolysaccharide and Hypoxia Potentiates Neoplastic Behavior and Risk for Prostate Carcinogenesis in Vivo. International Scholarly Research Notices, 2014, Article ID: 420429. http://dx.doi.org/10.1155/2014/420429

[12] Omabe, M., Ewenighi, C. and Onyeanusi, J.C. (2012) Increasing Evidence for Cigarette Smoking and Prostate Cancer Progression in Eastern Nigeria. International Journal of Biotechnology \& Biochemistry, 18, 61-69.

[13] Naber, K.G. and Weidner, W. (2000) Chronic Prostatitis-An Infectious Disease? Journal of Antimicrobial Chemotherapy, 46, 157-161. http://dx.doi.org/10.1093/jac/46.2.157

[14] Mishra, V.C., Allen, D.J., Nicolaou, C., Sharif, H., Hudd, C., Karim, O.M.A., Motiwala, H.G. and Laniado, M.E. (2007) Does Intraprostatic Inflammation Have a Role in the Pathogenesis and Progression of Benign Prostatic Hyperplasia? BJU International, 100, 327-331. http://dx.doi.org/10.1111/j.1464-410X.2007.06910.x

[15] Marignol, L., Coffery, M., Lawler, M. and Hollywood, D. (2008) Hypoxia in Prostate Cancer; a Powerful Shield against Tumor Destruction? Cancer Treatment Reviews, 34, 313-327. http://dx.doi.org/10.1016/j.ctrv.2008.01.006

[16] Ren, Q., Liang, J., Wei, J., et al. (2014) Epithelial and Stromal Expression of miRNAs during Prostate Cancer Progression. American Journal of Translational Research, 6, 329-339.

[17] Dennis, L., Lynch, C.F. and Tornes, J.C. (2002) Epidemiologic Association between Prostatitis and Prostate Cancer. Urology, 60, 78-83. http://dx.doi.org/10.1016/S0090-4295(02)01637-0 
[18] Sciarra, A., Di Silverio, F., Salciccia, S., Gomez, A.M.A., Gentilucci, A. and Gentile, V. (2007) Inflammation and Chronic Prostatic Diseases: Evidence for a Link? European Urology, 52, 964-972. http://dx.doi.org/10.1016/j.eururo.2007.06.038

[19] Davidsson, S., Fiorentino, M., Andrén, O., et al. (2011) Inflammation, Focal Atrophic Lesions, and Prostatic Intraepithelial Neoplasia with Respect to Risk of Lethal Prostate Cancer. Cancer Epidemiology, Biomarkers \& Prevention, 20, 2280-2287. http://dx.doi.org/10.1158/1055-9965.EPI-11-0373

[20] Omabe, M. and Nwobini, K.O. (2014) Deep Insight to Inflammation and Cancer. Atlas of Genetics and Cytogenetics in Oncology and Hematology, 18, 203-216.

[21] Höckel, M. and Vaupel, P. (2001) Biological Consequences of Tumor Hypoxia. Seminars in Oncology, 28, 36-41. http://dx.doi.org/10.1016/S0093-7754(01)90211-8

[22] Buter-worth, K.T., Mccarthy, H.O., Devlin, A., Ming, L., Robson, T., McKeown, S.R. and Worthington, J. (2008) Hypoxia Selects for Androgen Independent LNcap Cells with a More Malignant Genome and Phenotype. International Journal of Cancer, 123, 760-768. http://dx.doi.org/10.1002/ijc.23418

[23] Majmundar, A.J., Wong, W.J. and Simon, M.C. (2010) Hypoxia-Inducible Factors and the Response to Hypoxic Stress. Molecular Cell, 40, 294-309. http://dx.doi.org/10.1016/j.molcel.2010.09.022

[24] De Marzo, A.M., Marchi, V.L., Epstein, J.I. and Nelson, W.G. (1999) Proliferate, Inflammation Autrophy of the Prostate: Implication for Prostatic Carcinogenesis. American Journal of Pathology, 155, 1985-1992. http://dx.doi.org/10.1016/S0002-9440(10)65517-4

[25] Nelson, W.G., De marzo, A.M. and Isaacs, W.B. (2003) Prostate Cancer. The New England Journal of Medicine, 349, 366-381. http://dx.doi.org/10.1056/NEJMra021562

[26] Freedland, S.J. and Partin, A.W. (2006) Prostate Specific Antigen. Urology, 67, 458-460. http://dx.doi.org/10.1016/j.urology.2005.12.026

[27] Maier, G.S., Eberhardt, C., Strauch, M., Kafchitsas, K. and Kurth, A.A. (2014) Is Tartrate-Resistant Acid Phosphatase $5 \mathrm{~b}$ a Potent Bio-Marker for Late Stage Aseptic Implant Loosening? International Orthopaedics, 38, 2597-600. http://dx.doi.org/10.1007/s00264-014-2471-2 